César Merino-Soto ${ }^{1}$

Verónica López-Fernández 2,*

Paula Álvarez Merino ${ }^{3}$

Carmen Requena Hernández ${ }^{4}$

1. Profesor e investigador en el Instituto de Investigación de la Escuela de Psicología. Universidad de San Martín de Porres. Perú.

2. Doctora en Psicología y Ciencias de la Educación. Docente e investigadora en la Universidad Internacional de La Rioja. Logroño. La Rioja. España.

3. Doctora en Psicología y Ciencias de la Educación. Doctora e investigadora en la Universidad de León. León. España.

4. Doctora en Psicología. Docente e investigadora en la Universidad de León. León. España.

*Autor para correspondencia.

Correo electrónico: veronica.lopez@unir.net (Verónica López-Fernández).

\section{Efecto moderador de la creatividad en el rendimiento en memoria cotidiana en personas mayores}

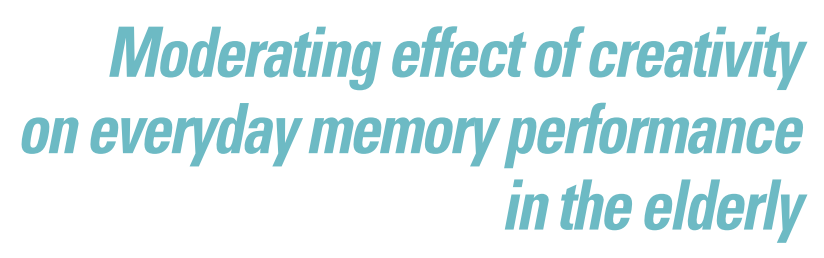

Recibido el 4 de marzo de 2019; aceptado el 11 de noviembre de 2019

\section{RESUMEN}

Antecedentes: la sociedad envejece a pasos agigantados y propone un reto importante al conjunto de profesionales, ya que una de las mayores preocupaciones en el transcurso de los ańos es la pérdida de funciones cognitivas como la memoria. Con base en ello, es relevante conocer qué estrategias pueden ayudar a preservar la capacidad mnésica de las personas mayores sanas. Objetivo: analizar el efecto moderador del constructo creatividad en la memoria cotidiana, así como el de la edad en una muestra de personas mayores sanas. Métodos: Participaron

106 personas mayores sanas y voluntarias provenientes de diferentes ciudades de España. Se les aplicó la prueba de creatividad CREA y la de memoria cotidiana RBMT. Resultados: La creatividad modera la pérdida de memoria en las personas mayores, en tanto que, por cada unidad de análisis de creatividad, aumenta la memoria. Lo contrario ocurre con la edad, al aumentar esta, la memoria disminuye. No se ha observado que la edad y la creatividad tengan un efecto de interacción conjunta sobre la memoria, actuando las dos por separado. Implicaciones: la creatividad puede ser incrementada en los programas de ocio y de intervención en psicoestimulación en el envejecimiento, ya que promover un contexto de actividades que fomenten la creatividad puede ser muy ventajoso para prevenir problemas de memoria en adultos mayores sanos. Se necesitan

más estudios que ahonden en esta relación y el tipo de actividades concretas creativas que pueden ser beneficiosas y efectivas.

PALABRAS CLAVE: Envejecimiento, creatividad, memoria, reserva cognitiva.

\section{ABSTRACT}

Background: The society ages at a rapid pace proposing an important challenge to the group of professionals, since one of the biggest concerns over the years is the loss of cognitive functions such as memory. Based on this, it is relevant to know what strategies can help to preserve the mnemonic capacity of healthy elderly people. Objective: to analyze the moderator effect of the creativity construct in the daily memory, as well as the age effect in a sample of healthy elderly people. Methods: 106 healthy and voluntary elderly people from different cities of Spain participated. The CREA creative test and the RBMT daily memory test were applied. Results: Creativity moderates memory loss in older people, while for each unit of creativity analysis, memory increases. The opposite happens with age, as it increases, memory decreases. It has not been found that age and creativity have a joint interaction effect on memory, acting both separately. Implications: creativity can be increased in leisure programs and intervention in psychostimulation in aging, since promoting a context of activities that promote creativity can be very advantageous to prevent memory problems in healthy older adults. More studies are needed that deepen this relationship and the type of concrete creative activities that can be beneficial and effective.

KEYWORDS: Aging, creativity, memory, cognitive reserve.

\section{- INTRODUCCIÓN}

No cabe duda de que nos encontramos inmersos en el gran desafío que supone el envejecimiento poblacional, debido fundamentalmente al aumento de la esperanza de vida y a la disminución de la natalidad ${ }^{1}$. En este sentido, los profesionales debemos aportar estrategias que faciliten la superación de dicho reto posibilitando el bienestar de las personas en su proceso de envejecimiento, en aras de lograr un envejecimiento sano, exitoso y de calidad. Uno de los aspectos más relevantes en este desafío demográfico y uno de los que más preocupa a las personas es la conser- vación de las funciones cognitivas a medida que se envejece, como es el caso concreto de la memoria ${ }^{2}$.

Es esta línea, la creatividad ha mostrado ser beneficiosa en el envejecimiento exitoso ${ }^{3}$ en cuanto a que ayuda a conservar la salud mental, las funciones cognitivas e incluso fisiológicas ${ }^{4}$. La creatividad es un constructo que se relaciona con múltiples dominios de la actividad humana tanto cognitivos como afectivos o motivacionales ${ }^{5}$. Sin embargo, a pesar de su relevancia, su importancia en el envejecimiento y la prevención de deterioro no se explicita en la literatura científica, aunque hay estudios que muestran que es importante incluir el aprendizaje a lo largo de la 
vida con estrategias creativas ${ }^{6}$, y que la creatividad verbal se relaciona con la reserva cognitiva y cerebral, siendo incluso un posible indicador de esta ${ }^{7}$.

Teniendo esto en cuenta, el presente estudio pretende contribuir a esclarecer el papel de la creatividad en el proceso de envejecimiento en relación con la memoria, de tal forma que el objetivo es evaluar el papel moderador de la creatividad como una variable naturalmente presente, de la relación entre la edad y la memoria, para identificar la posible influencia entre ellas. El tipo de memoria investigada aquí es la memoria cotidiana; y la creatividad cuantificada aquí se caracteriza principalmente por evaluar la capacidad del sujeto para formular preguntas a partir de una imagen elicitadora. Ambas variables se recogieron mediante instrumentos estandarizados y psicométricos. Conocer el denominado efecto moderador ayuda a reducir la probabilidad de obtener conclusiones sesgadas sobre la relación entre dos variables $^{8}$, porque distingue un gradiente de intensidad del moderador (variable $z$ ) que condiciona la relación entre dos variables, es decir entre la variable predictora (o independiente, usualmente etiquetada con $x$ ) y la variable criterio (o dependiente, usualmente etiquetada con $y)^{9}$. Por lo tanto, el efecto moderador se define como una relación entre dos variables (independiente o exógena, y dependiente o endógena), condicionada por los niveles de otra variable (moderador), esto es, que la relación entre ambas depende o está en función del moderador ${ }^{9,10}$. La detección del efecto moderador de una variable habitualmente se representa por el término de interacción en una ecuación de regresión múltiple: $y=b 0+b 1 x+b 2 z+b 3 x z$, donde $y$ es la variable criterio, $x$ es la variable predictora, y $z$ es el moderador; el término de interacción es $x z$.

En el contexto del presente estudio, es plausible que el efecto habitual de la edad sobre la memoria varíe de acuerdo con diferentes niveles de creatividad que el adulto mayor ha desarrollado. Concretamente, el efecto moderador de la creatividad, tal como es medido en el presente estudio, consiste en que la usual asociación negativa (decremento) de la edad sobre la memoria puede variar cuando el sujeto aplica o ha desarrollado bajos o altos niveles de creatividad.

\section{- MÉTODO}

\section{Diseño}

Se trata de un diseño transversal descriptivo y analítico.

\section{Participantes}

La muestra estuvo compuesta por 106 participantes mayores de 60 años, procedentes de diferentes regiones de España; 39 de ellos varones (36,8\% del total). El promedio del grupo fue $71,7(\mathrm{DE}=5,5)$, y varió entre 60 y 86 ańos. Las diferencias de edad respecto al sexo fueron triviales y estadísticamente no significativas y de tamaño pequeño, $t(104)$ $=0,601, p=0,54, d=0,121$. Los criterios de inclusión para poder participar en el estudio fueron: ser mayor de 60 años, no tener diagnóstico de deterioro cognitivo (evaluado a través del Minimental Test: MMSE > 25) y aportar un consentimiento informado cumplimentado y firmado de participación voluntaria en el estudio. Criterio de exclusión: historial de enfermedades psiquiátricas y neurológicas. Respecto al nivel educativo, el conjunto de la muestra presentaba estudios primarios $(87,27 \%)$, secundarios $(5,46 \%)$ y universitarios $(7,27 \%)$. En cuanto a la ocupación desempeñada en su etapa laboral, el $6 \%$ pertenece al sector primario, el $24 \%$ al secundario y el 26\% al terciario. El 44\% restante son mujeres con dedicación exclusiva a las tareas del hogar de manera no remunerada.

\section{Instrumentos}

Para evaluar la creatividad se empleó la prueba CREA: inteligencia creativa ${ }^{11}$. En concreto se administró la lámina $C$, empleada en personas mayores de 55 años en un estudio previo ${ }^{12}$. El test CREA ha mostrado alta fiabilidad y validez ${ }^{13}$. CREA consiste en mostrar material gráfico al evaluado y durante 4 minutos la persona debe formular preguntas respecto a lo que está viendo. Se obtiene una única puntuación global de creatividad ${ }^{11}$

En cuanto a la memoria, se utilizó el Test conductual de memoria Rivermead $(\text { RBMT })^{14}$. Se trata de una prueba que evalúa aspectos verbales, visuales, recuerdo y reconocimiento de la memoria cotidiana a través de diferentes subpruebas y que permite obtener una medida general. La fiabilidad de la prueba en cuanto a la puntuación global alcanza un valor elevado $(0,87)$ según informan los autores ${ }^{14}$.

\section{Procedimiento}

Recolección de datos. La muestra se reclutó entre 450 usuarios de un balneario situado en Salamanca (España). La captación de los participantes se realizó mediante una charla informativa en la que se presentó el estudio. Los voluntarios interesados cumplimentaron una ficha con su nombre, edad y un teléfono de contacto a través del que se concretó la cita para la evaluación una vez dado su consentimiento.

Los instrumentos de evaluación fueron aplicados siguiendo las instrucciones que se especifican en los manuales de ambas pruebas, con un tiempo de administración en RBMT de 30 minutos y en CREA de 15 minutos. Estas pruebas se aplicaron de forma alterna e individual por dos de los investigadores que llevaron a cabo el estudio en una sala libre de distracciones.

Análisis. Antes de efectuar el análisis principal, se evaluaron varias condiciones propias del análisis de moderación que fortalecen la validez interna de los resultados. Primero, se identificaron posibles outliers multivariados ${ }^{15}$, mediante la distancia $D^{2}$ de Mahalanobis ${ }^{16}$; para ello se aplicó un programa ad hoc ${ }^{17}$. Segundo, se exploraron las relaciones lineales entre el predictor (edad), el moderador (creatividad) y el criterio (memoria); para esto se utilizó el comando medias del programa IBM SPSS 25. Finalmente, para probar las presunciones conjuntas del modelamiento lineal (linealidad, heterocedasticidad, independencia y normalidad), se implementó una prueba global $\chi^{2}(\mathrm{gl}=4)$ respecto a estas presunciones ${ }^{18}$, en que la hipótesis nula fue el cumplimiento conjunto de esas presunciones. Este método fue implementado mediante el programa gvlm $^{18}$.

Con respecto al análisis de moderación y de acuerdo con el objetivo del estudio, se identificaron la edad, la creatividad y la memoria, respectivamente, como variable predictora, variable moderadora y variable de efecto. En primer lugar, para reducir problemas de multicolinealidad debido a la introducción del término de interacción (es decir, el efecto de moderación, $b 3 x z$ ), se centraron las variables correspondientes al predictor (edad) y moderador (creatividad), de tal modo que estas variables centradas tendrían sus medias iguales a cero ${ }^{9}$. Para la significación estadística, se aplicó el procedimiento bootstrap con 5000 muestras para estimar el error estándar y los coeficientes b. Para obtener la magnitud del efecto moderador se utilizó la diferencia de $R^{2}\left(\Delta_{R}^{2}\right)$ y $f$ (según informan Helm y Mark ${ }^{19}$ ) entre el modelo sin moderador y con moderador. Todo el análisis de moderación se efectuó mediante el programa PROCESS ${ }^{20}$. 
Tabla 1. Estadísticos descriptivos y correlacionales de las variables de estudio

\begin{tabular}{|c|c|c|c|c|c|c|c|c|c|c|}
\hline & \multicolumn{7}{|c|}{ Estadísticos descriptivos } & \multicolumn{3}{|c|}{ Correlaciones } \\
\hline & M & $\mathrm{DE}$ & Mín. & Máx. & $\overline{Z_{\mathrm{b} 2}}$ & $Z_{\mathrm{b} 1}$ & $K^{2}$ & Edad & Creatividad & Memoria \\
\hline Edad & 71,61 & 5,452 & 60 & 86 & 0,384 & 3,093 & 9,719 & 1 & & \\
\hline Creatividad & 2,09 & 1,927 & 0 & 7 & 0,277 & 3,145 & 9,968 & $-0,097$ & 1 & \\
\hline Memoria & 6,09 & 3,363 & 0 & 12 & $-1,995$ & $-2,620$ & 10,850 & $-0,225^{a}$ & $0,450^{\mathrm{a}}$ & 1 \\
\hline
\end{tabular}

Nota. Creatividad: medida por la puntuación total del instrumento CREA; Zzz: valor estandarizado de la curtosis; Z Zbi: valor estandarizado de la asimetría; K': coeficiente de normalidad D'Agostino-Pearson. ${ }^{a} p<0,05$.

\section{- RESULTADOS}

\section{Análisis preliminar}

Valores extremos. En el nivel de significación igual a $05(F[3,102]=$ 16,63 , un participante fue identificado como outlier multivariado mediante la distancia Mahalonobis más grande (Mahalonobis- $\left.\mathrm{D}^{2}=18,34\right)$ respecto a los 10 sujetos con distancias más grandes (de 6,12 a 18,34); para reducir la sensibilidad de este outlier en el contexto del presente tamaño muestral, este participante fue eliminado en los datos; por lo tanto, la muestra de análisis se redujo a 105 participantes.

Linealidad. Se evaluó la linealidad de la relación entre la edad y las variables memoria y creatividad tomados independientemente. En la relación edad-memoria, la linealidad fue detectada $(F[1,84]=5,025, p=$ $0,028)$, y el desvío de la linealidad no superó el error de muestreo $(F[20$, $84]=0,782, p=0,072)$. Respecto a la relación edad-creatividad, la linealidad $(F[1,84]=0,016, p=0,90)$ y el desvío de la linealidad $(F[20$, $84]=0,997, p=0,47)$ entre ellas no fue mayor que el error de muestreo. Estos resultados indican que la linealidad está presente en las relaciones entre las variables. En la tabla 1 se muestran los estadísticos básicos de las variables de estudio.

Información descriptiva y correlacional. Las variables predictoras muestran entre fuerte y moderada asimetría, mientras su curtosis se mantiene similar a como ocurre en la distribución normal (tabla 1). En conjunto, la distribución de las puntuaciones no tiene la forma de la distribución normal. Se observa que la relación entre la edad y la puntuación de memoria puede considerarse pequeña, y la relación entre memoria y creatividad es moderada; la dirección de la covariación lineal entre ellas (negativa y positiva, respectivamente) es teóricamente respaldada.

Presunciones. La prueba global del cumplimiento simultáneo de las presunciones fue estadísticamente significativa $\left(\chi^{2}=17,233, p<0,01\right)$, indicando que los datos no cumplieron alguna de estas presunciones. Examinando con más detalle, se halló que mientras que la asimetría $\left(\chi^{2}\right.$ $=1,621)$, curtosis $\left(\chi^{2}=1,070\right)$ y linealidad $\left(\chi^{2}=2,168\right)$ fueron estadísticamente no significativas $(p>0,13)$, esto es, que los datos cumplieron con estas condiciones, la ausencia de heterocedasticidad no se cumplió $\left(\chi^{2}=12,375, p<0,01\right)$. Para atenuar algún error metodológico (error Tipo I o II) debido a este problema, se utilizó un enfoque robusto para estimar la matriz de covarianzas y los errores estándar de los coeficientes beta, mediante el método HC3 de Davidson y MacKinnon ${ }^{21}$; este es altamente recomendado para atenuar el efecto de la heterocedastici$\mathrm{dad}^{22-24}$.

Análisis de moderación. Los resultados obtenidos del bootstrap (no mostrados aquí) fueron prácticamente indistinguibles de las estimaciones asintóticas de los coeficientes b y sus intervalos de confianza, por lo que, para presentar de la forma más sencilla posible los resultados, únicamente se exponen los resultados asintóticos. El modelo final explicó el
$23,89 \%$ de la varianza $(R=0,488)$ y fue estadísticamente significativo en ambos estimadores robustos, $F(3,101)=12,873, p<0,01$. En la tabla 2 aparece la información de los parámetros estimados, donde la edad y la creatividad (medida por CREA) influyeron sobre la memoria más allá del error de muestreo. En relación con la edad, para cada incremento de un ańo, la memoria decrementa en 0,113 puntos; en dirección contraria, por cada unidad de incremento en la puntuación de CREA, la memoria se incrementa en 0,764 puntos. Por otro lado, la inclusión del término de interacción no fue estadísticamente significativa, F-HC3 $(1,101)=0,393$; y la magnitud de su efecto fue trivial.

Tabla 2. Resultados del análisis de moderación

\begin{tabular}{|l|c|c|c|c|c|}
\multirow{2}{*}{} & \multirow{2}{*}{$\mathbf{b}$} & \multicolumn{2}{|c|}{$\begin{array}{c}\text { IC95\% } \\
\text { (coeficiente b) }\end{array}$} & \multirow{2}{*}{$\begin{array}{c}\text { e.e } \\
\text { (HC3) }\end{array}$} & \multirow{2}{*}{$\boldsymbol{t}$} \\
\cline { 3 - 5 } & & Inf. & Sup. & & \\
\hline Constante & 6,064 & 5,4562 & 6,6732 & 0,306 & $19,769^{b}$ \\
\hline Edad & $-0,113$ & $-0,2210$ & $-0,0063$ & 0,054 & $-2,099^{a}$ \\
\hline CREA & 0,764 & 0,4408 & 1,0890 & 0,163 & $4,681^{b}$ \\
\hline Edada CREA & $-0,020$ & $-0,0862$ & 0,0448 & 0,033 & $-0,627$ \\
\hline$F(3,101)$ & 12,873 & & & & \\
\hline$\Delta_{R}^{2}$ & 0,0035 & & & & \\
\hline$f$ & 0,0045 & & & & \\
\hline
\end{tabular}

Nota. e.e.: error estándar del coeficiente b; HC3: ajuste de la matriz de covarianzas y error estándar (ver texto); $t$. prueba estadística.

${ }^{\mathrm{a}} p<0,05 \cdot{ }^{\mathrm{b}} p<0,01$.

\section{- DISCUSIÓN}

El presente estudio aporta resultados relevantes respecto a que cuantifica el efecto de la moderación de la edad sobre la memoria y también de la creatividad sobre la memoria, explicitando la importancia del pensamiento creativo en las funciones mnésicas. De acuerdo con los resultados, la creatividad no tiene un efecto moderador importante sobre la usual relación de la edad y el decremento en la habilidad mnésica. Esto es, una fuerte o débil habilidad creativa no parece producir cambios importantes en el decremento de la memoria, pero sí efectos pequeńos. Por otro lado, la creatividad de manera independiente tiene un efecto en la disminución de la memoria, esto es, incrementa esta habilidad. Este efecto, como reportamos anteriormente, no condiciona la relación edad - memoria.

Estos resultados junto con otros que muestran que la creatividad puede optimizar la memoria podría indicarnos que la creatividad puede ser un componente de la reserva cognitiva ${ }^{7}$. Las implicaciones prácticas 
en este sentido nos sitúan en la necesidad de que los programas de psicoestimulación en el envejecimiento incluyan actividades creativas, ya que promover un contexto de actividades que promuevan la creatividad puede ser muy ventajoso para prevenir problemas de memoria en adultos mayores sanos. Una forma de incrementar dichos programas de prevención puede ser a través de las actividades de ocio en actividades cotidianas ${ }^{25}$.

Se necesitan más estudios que ahonden en esta relación y el tipo de actividades concretas creativas que pueden ser beneficiosas y efectivas, así como la duración de estas en el proceso de envejecimiento.

Otro aporte interesante de este estudio es haber seguido la recomendación de otros autores de ampliar el número de casos de evaluación de la creatividad aplicando la lámina $\mathrm{C}$ de la prueba CREA en mayores de 55 ańos por ser la que menos se ha empleado en personas mayores ${ }^{12}$.

Futuros estudios podrían comparar diferentes grupos etarios ${ }^{6}$, pero en este sentido, una dificultad añadida sería que no existen instrumentos que evalúen la creatividad en el envejecimiento de forma específi$\mathrm{ca}$, aunque sí ha habido aplicaciones generales a muestra de personas mayores que evidencian bajas puntuaciones, debidas quizás a menor creatividad en personas mayores o, por otra parte, por emplear instrumentos no apropiados ${ }^{26}$. Para esclarecer este punto, un primer paso sería adaptar diferentes pruebas y validarlas específicamente en esta población, por ejemplo, tratando de incluir factores de corrección o permitiendo la respuesta verbal en lugar de por escrito, como se permite en edades concretas en la población de menor edad ${ }^{11}$, y analizar si los resultados sufren o no variaciones en el efecto de moderación, pero también que sirvan para ser más fiables y válidas al valorar la efectividad del uso de programas creativos que puedan estimular la creatividad.

Por último, sería interesante complementar con medidas de actividad cerebral, como la electroencefalografía, estudios de estas características, ya que no existen estudios de creatividad en personas mayores con electroencefalografía que ahonden en la relación compleja entre memoria y creatividad $^{27}$.

\section{- CONCLUSIONES}

El presente estudio ha evaluado el papel moderador de la creatividad en la relación entre la memoria y la edad, hallándose que la creatividad no tiene un efecto moderador importante sobre la relación de las variables mencionadas. No obstante, la creatividad de manera independiente sí tiene un efecto en el incremento de la memoria

\section{Conflicto de intereses}

Los autores declaran no tener ningún conflicto de intereses.

\section{Fuente de financiación}

La presente investigación no ha recibido ayudas específicas provenientes de agencias del sector público, sector comercial o entidades sin ánimo de lucro.

\section{BIBLIOGRAFÍA}

1. Berg S, Dahl A, Nilsson S. Declive cognitivo y demencia. En: Fernández-Ballesteros $\mathrm{R}$, ed. PsicoGerontología: perspectivas europeas para un mundo que envejece. Madrid: Pirámide; 2009. p. 223-42.

2. Tabloski P. Gerontological nursing. Upper Saddle River, NJ: Pearson Education; 2006.

3. Fisher BJ, Specht DK. Successful aging and creativity in later life. J Aging Stud. 2000;13(4):457-72

4. Flood M. Phillips KD. Creativity in older adults: A plethora of possibilities. Issues Ment Health Nurs. 2007;28:389-411.

5. Gonen-Yaacovi G, de Souza LC, Levy R, Urbanski M, Josse G, Volle E. Rostral and caudal prefrontal contribution to creativity: a meta-analysis of functional imaging data. Front Hum Neurosci. 2013;7:465

6. Burgos C, Osses S. Creatividad y neurociencia: binomio esencial en el contexto educativo. Rev. méd. Chile. 2015;142:948-9.

7. Palmiero M, Di Giacomo D, Passafiume D. Can creativity predict cognitive reserve? J Creative Behav. 2016;50(1):7-23.

8. Helm R, Mark A. Analysis and evaluation of moderator effects in regression models: state of art, alternatives and empirical example. Rev Manag Sci. 2012;6(4):307-32.

9. Mackinnon DP, Luecken LJ. How and for whom? Mediation and Moderation in Health Psychology. Health Psychology. 2008:27(2):99-102

10. Cohen J, Cohen P, West SG, Aiken LS. Applied multiple regression/ correlation analyses for the behavioral sciences. 3. ${ }^{\text {a }}$ ed. Mahwah, New Jersey: Lawrence Erlbaum; 2003.
11. Corbalán FJ, Martínez F, Donolo D, Tejerina M, Limiñana RM. CREA: Inteligencia Creativa. Una medida cognitiva de la creatividad. Madrid: TEA Ediciones; 2003.

12. Elisondo R, Donolo D. Contextos y creatividad. Variables sociodemográficas y datos normativos en el Test CREA. Revista Evaluar. 2018;18(3):14-29. Recuperado de https://revistas. unc.edu.ar/index.php/revaluar [consultada el 19 de febrero de 2019].

13. Martínez F. Característica psicométricas del CREA (inteligencia creativa). Un estudio con población española y argentina. RIDEP 2003:16(2);71-83.

14. Wilson B, Cockburn J, Baddeley AD. The Rivermead Behavioural Memory Test. Reading, UK: Thames Valley Test Co.; 1985.

15. Johnson RA, Wichern DW. Applied Multivariate Statistical Analysis. New Jersey: Prentice Hall; 2002.

16. Mahalanobis PC. On the generalized distance in statistics. National Institute of Science of India; 1936.

17. DeCarlo LT. On the meaning and use of kurtosis. Psychological Methods. 1997:2:292-307.

18. Pena EA, Slate EH. Gvima: Global Validation of Linear Models As sumptions. R package version 1.0.0.3.; 2019. https://CRAN.R-project org/package=gvlma [consultado 13 de febrero de 2019].

19. Helm R, Mark A. Analysis and evaluation of moderator effects in regression models: state of art, alternatives and empirical example. R Manag Sci. 2012;6(4):307-32.
20. Hayes AF. Introduction to mediation, moderation and conditional process analysis. 2. ${ }^{\text {a }}$ ed. New York: The Guilford Press; 2018.

21. Davidson R, MacKinnon JG. Estimation and inference in econometrics. Oxford: Oxford University Press; 1993.

22. Long JS, Ervin LH. Using heteroskedasticity consistent standard errors in the linear regression model. American Statistician. 2003;54:217-24.

23. Rosopa PJ, Schaffer MM, Schroeder, AN. Managing heteroscedasticity in general linear models. Psychological Methods. 2013:18(3):335-51.

24. Hayes AF, Cai L. Using heteroskedasticity-consistent standard error estimators in OLS regression: An introduction and software implementation. Behav. Res. Methods. 2007;39(4):709-722.

25. López V, Requena C. El ocio mejora la creatividad y el funcionamiento cerebral de personas mayores sanas con y sin deterioro cognitivo. I congreso Internacional de Psicología, salud y Educación; 2017 Nov 8-11; Oviedo, España.

26. Ferrándiz C, Ferrando M, Soto G, Sáinz M, Prieto MD. Divergent thinking and its dimensions: what we talk about and what we evaluate? An. de Psicología. 2017;33(1):40-7.

27. López V, Requena C, Álvarez P. Creatividad y plasticidad cerebral en mayores sanos. Un análisis basado en la actividad cerebral. Avances en ciencias de la educación y del desarrollo. 4th International Congress of Educational Sciences and Development; 2016; 236-42. 